\title{
Das Modellprojekt „Entwöhnung im Verbundsystem (EVS)“: Ein Beitrag zur Verbesserung von Kooperationen in der Suchtkrankenhilfe
}

Knut Tielking
Gabriele Kuß

\author{
The Model Project "EVS": Added Improvement of Cooperation in \\ Addiction Treatment
}

\section{Zusammenfassung}

Im Rahmen des Modellprojekts „Entwöhnung im Verbundsystem (EVS)“1 werden derzeit innovative Ansätze zur Verbesserung von Kooperationen in der Suchtkrankenhilfe erprobt. Wesentliche Elemente zur Verbesserung der Kooperationsbeziehungen sind die Arbeit in einem so genannten Qualitätszirkel und speziell entwickelte Kooperationsstandards. Auf dieser Basis sind in den ersten sechs Monaten des Modellprojekts bereits ein deutlich veränderter Leistungsrahmen (u.a. Leistungszusage für ambulant-stationäre Behandlungselemente in einem Zeitraum von 52 Wochen), ein therapeutisches Gesamtkonzept zwischen fünf ambulanten und drei stationären Leistungsanbietern unterschiedlicher Träger und eine Kooperationsvereinbarung verabschiedet worden. Der Artikel skizziert das Vorhaben und zeigt anhand der Matrix „Kooperationsstandards“ einen Weg zur Strukturierung der Vielzahl von Aspekten, die in Kooperationen eine Rolle spielen können, auf.

\section{Schliuisselwörter}

Vernetzung · Medizinische Rehabilitation · Ambulant-stationäre Kombinationsbehandlung · Kooperationsstandards · Qualitätszirkel

\section{Abstract}

In the context of the model project „Drug Withdrawal in an Integrated System (EVS)“2 new approaches to an advancement of cooperations between providers of health care services for addicted persons are currently tested. Establishing so-called quality circles and developing cooperation standards have been identified as particularly important for improving cooperative relations. Working on this principle it was possible in the first six months of the project to achieve a significant extension of the healthcare services funded (among other things a generalized confirmation to provide funds for specific outpatient/inpatient treatments within a 52-week time frame). Additionally a joint therapeutic concept of five outpatient and three inpatient service providers - financed by different agencies - as well as a cooperation agreement were adopted. The article gives an outline of the project and uses the matrix of "cooperation standards" to show how the large variety of aspects governing cooperation agreements can be structured.

\section{Key words}

Networking · Medical Rehabilitation · Combined Outpatients/ Inpatient Treatment · Cooperation Standards · Quality Circles

Anmerkung

${ }^{1}$ Das Modellprojekt ist auf drei Jahre angelegt und wird finanziell von der Landesversicherungsanstalt Oldenburg-Bremen gefördert.

${ }^{2}$ The model project has been planned to run for three years and is funded by the Oldenburg-Bremen State Insurance Company.

Institutsangaben

Carl von Ossietzky Universität Oldenburg

Korrespondenzadresse

Dr. Knut Tielking · Carl von Ossietzky Universität Oldenburg

Institut für Politikwissenschaft II - Fachbereich 3 • Postfach $2503 \cdot 26111$ Oldenburg .

E-mail: alkohol@uni-oldenburg.dewww.uni-oldenburg.de/saus 
An diesem Punkt setzt das Modellprojekt „Entwöhnung im Ver-

Vernetzung, Verbundsysteme, Kooperation, Koordination - das sind in vielen gesellschaftlichen Bereichen die Schlagworte unserer Zeit. Nicht nur in betriebswirtschaftlichen Überlegungen zur Organisationsentwicklung, sondern auch im Gesundheitswesen führten Überlegungen zur Gesundheitsförderung durch systematische Organisationsentwicklung zur Suche nach neuen, wirksamen Kooperationsformen in größeren sozialen Zusammenhängen [1].

Auch für erfolgreiche Suchtkrankenhilfe ist es von grundlegender Bedeutung, dass einrichtungsübergreifend ein möglichst breites Spektrum von Therapieformen (ambulant, teilstationär, stationär), Therapierichtungen (psychoanalytische, verhaltenstherapeutische, integrative Orientierungen) und Therapieangeboten (psychotherapeutische, medizinische, soziotherapeutische) vorgehalten wird. Dies erfordert eine Vielzahl unterschiedlicher Hilfen, die interdisziplinär ausgestaltet werden, gleichzeitig aber zur Verzahnung der einzelnen Behandlungselemente formell ausgestaltete Kooperationen zwischen Leistungsanbietern wie auch mit Leistungsträgern notwendig machen. In diesem Bereich der Kooperation von Einrichtungen/ Personen und Koordinierung von Hilfsmaßnahmen existieren bisher jedoch Defizite im Suchtkrankenhilfesystem, die eine effiziente Nutzung vorhandener Ressourcen einschränken [2 -9].

Auch Ergebnisse einer Studie, die die Arbeitsstelle „Sucht- und Drogenforschung (SAUS)“ der Universität Oldenburg in der Region Oldenburg-Bremen durchführte (Laufzeit 1996 bis 2000), bestätigen, dass in der Zusammenarbeit verschiedener Institutionen/Personen im Rehabilitationsprozess, eine Optimierung durch eine engere Abstimmung von Maßnahmen speziell zwischen den stationären und ambulanten Leistungsanbietern möglich ist [9]. Die konzeptionell von den Leistungsanbietern beschriebene Notwendigkeit und Sinnhaftigkeit von Kooperationen bis hin zu Verbundsystemen kann derzeit im Praxisalltag oft nicht zufriedenstellend umgesetzt werden. Kontakte zwischen ambulanten und stationären Therapeuten finden nicht, wie theoretisch angestrebt, persönlich statt, sondern, wenn überhaupt, nur telefonisch; bei ca. jedem zehnten Patienten/Klienten gab es sogar überhaupt keine Kontakte.

Natürlich gibt es Gründe, die eine optimale Kooperation im Alltag erschweren. Dies sind z.B. die große Zahl der ambulanten Einrichtungen mit denen Kliniken in Kontakt stehen, z.T. große Entfernungen zwischen Kliniken und ambulanten Einrichtungen und häufig zu geringe personelle Kapazitäten der Einrichtungen. Diese Problematiken machen einerseits deutlich, dass die theoretisch anerkannte Notwendigkeit einer engen Zusammenarbeit in der Praxis auf Grenzen stößt. Andererseits gibt es positive Ansätze im Rahmen informeller Kooperationen, die im Praxisalltag auf- und ausgebaut wurden, bis hin zu ausgearbeiteten Konzepten einiger Einrichtungen, die bereits in Modellversuche münden [3]. Somit sind Überlegungen zu einer verstärkten Kooperation zwischen stationären und ambulanten Leistungsanbietern zu differenzieren bzw. Standards für die Suchtkrankenhilfe $[10,11] \mathrm{zu}$ erarbeiten und weiterzuentwickeln, die Argumentationshilfen für die Diskussion und insbesondere für die praktische Arbeit bieten. bundsystem (EVS)“ an: Es soll zur Verbesserung der Kooperationsstrukturen in einem notwendigerweise einzugrenzenden Teilbereich des Suchtkrankenhilfesystems beitragen: dem Bereich der medizinischen Rehabilitation. Die Ausweitung von Innovationskonzepten zum Thema Vernetzung auf andere Sektoren der Suchthilfe ist langfristig unabdingbar, da hier insgesamt großer Veränderungsbedarf besteht [9]. Nachfolgend werden die innovativen Ansätze des Projekts vor und Empfehlungen zur Verbesserung von Kooperation zur Diskussion gestellt.

\section{Ziele des Modellprojekts}

Das Modellprojekt EVS soll die Individualisierung und Flexibilisierung der Behandlung bei gleichzeitig verbindlicher Festlegung der arbeitsteiligen Aufgaben zwischen Leistungsanbietern fördern. Dabei stehen folgende Ziele im Mittelpunkt der dreijährigen Laufzeit (2000-2003):

1. die stärkere Nutzung ambulanter Leistungen,

2. Auf- und Ausbau kombiniert ambulant-stationärer Rehabilitationsleistungen und

3. die Entwicklung verbindlicherer Formen der Zusammenarbeit zwischen ambulanten und stationären Leistungsanbietern bei der Rehabilitation von Alkoholabhängigen,

4. die Verbesserung der Zusammenarbeit zwischen Leistungsanbietern und Leistungsträgern.

\section{Besonderheiten des Modellprojekts}

Stellt man die medizinische Rehabilitation als wesentliches Element innerhalb des Versorgungssystems für Alkoholabhängige in den Mittelpunkt der Gesamtbehandlung, sind zwei Kooperationsbereiche besonders zu betrachten: Zum einen wird den Übergängen - häufig auch als „Schnittstellen“ bezeichnet - zwischen dem Rehabilitationssystem für Alkoholabhängige und dem übrigen Hilfesystem (z. B. Hausärzte, Krankenhäuser) eine ausschlaggebende Bedeutung für den Erfolg der Gesamtbehandlung zugeschrieben; zum anderen werden insbesondere die „Schnittstellen“ innerhalb des Rehabilitationssystems, d.h. zwischen ambulanten und stationären Leistungsanbietern, als wesentlich für den Erfolg des Rehabilitationsprozesses und damit auch für die Gesamtbehandlung eingeschätzt. In beiden Bereichen sollen durch eine bedarfsgerechte Koordination aller Versorgungsdienste dem Suchtkranken entsprechend seiner individuellen Behandlungsbedürfnisse flexible Übergänge von einer Betreuungs- oder Behandlungsform in eine andere ermöglicht werden. Dies erfordert im Anspruch eines optimalen Ineinandergreifens der Hilfen eine entsprechende Ausgestaltung hinsichtlich „Kooperation“ und „Koordination“ zwischen den Leistungsanbietern, aber auch mit den zuständigen Kosten-/ Leistungsträgern, die einzelne Maßnahmen bewilligen und finanzieren.

Umso wichtiger ist es, eine Entwicklung voranzubringen, die von den derzeit separaten Institutionen zu einer neuen Ausrichtung der medizinischen Rehabilitation auf gemeinsame Inhalte und Organisationsformen zwischen ambulanten und stationären Leistungsanbietern führt. Um eine Vernetzung in diesem Sinne zu erreichen, bedarf es der Definition der besonderen Merkmale 
ambulanter und stationärer Rehabilitation. Diese ist auf der Ebene der Kosten-/Leistungsträger in diversen Empfehlungs- und Gesamtvereinbarungen vorgenommen worden. Im Zusammenhang mit Überlegungen zur Verbesserung der Kooperation bedarf es aber auf der Ebene der Leistungsanbieter weitergehender Abstimmungen und Konkretisierungen, die in Kooperationsabsprachen, -vereinbarungen oder in gemeinsamen Therapiekonzepten münden sollten, um über ein Modell der reinen Koexistenz hinauszukommen. Speziell auf ambulant-stationäre Zusammenarbeit bezogen, fasst Abb. 1 wesentliche Ebenen zusammen, die bei der Organisation von Verbundsystemen wesentlich sind und der konkreteren Ausgestaltung bedürfen (siehe „Innovation Kooperationsstandards“).

Von großer Bedeutung für das Modellprojekt EVS war zunächst die Flexibilisierung des Leistungsrahmens (vgl. Behandlungsvarianten Abb. 2) durch den beteiligten Leistungsträger, die LVA Oldenburg-Bremen. Innerhalb der gesetzlichen Vorgaben, insbesondere des SGB VI und des RehaAnglG, obliegt es den Leistungsträgern, durch ein entsprechendes Antragsverfahren eine Flexibilisierung der Leistungsverläufe zu unterstützen. ${ }^{3}$
Darüber hinaus erfordert das Projekt auf der Seite der Leistungsanbieter eine eindeutige Definition von Zuständigkeiten und Verbindlichkeiten. Orientierungshilfe bieten bei der Erarbeitung derartiger Kooperationsbeziehungen so genannte Kooperationsstandards [13], auf die aufgrund ihrer Bedeutung im nächsten Abschnitt ausführlicher eingegangen wird.

Formelle Grundlagen des Verbundmodells EVS sind:

- ein therapeutisches Gesamtkonzept zwischen den beteiligten ambulanten Beratungs- und Behandlungsstellen sowie den Kliniken und

- eine Kooperationsvereinbarung zwischen diesen Leistungsanbietern und darüber hinaus den beteiligten Einrichtungsträgern. ${ }^{4}$

Zur Förderung der institutionellen und personellen Kooperation wird im Rahmen von EVS ein Qualitätszirkel als steuerndes Gremium eingesetzt. Der Qualitätszirkel schafft eine formelle Basis und beteiligt Vertreter der Landesversicherungsanstalt Oldenburg-Bremen, drei stationärer und fünf ambulanter Leistungsanbieter; die Moderation und wissenschaftliche Begleitung obliegt der Universität Oldenburg. Relevante Themen und Prob-
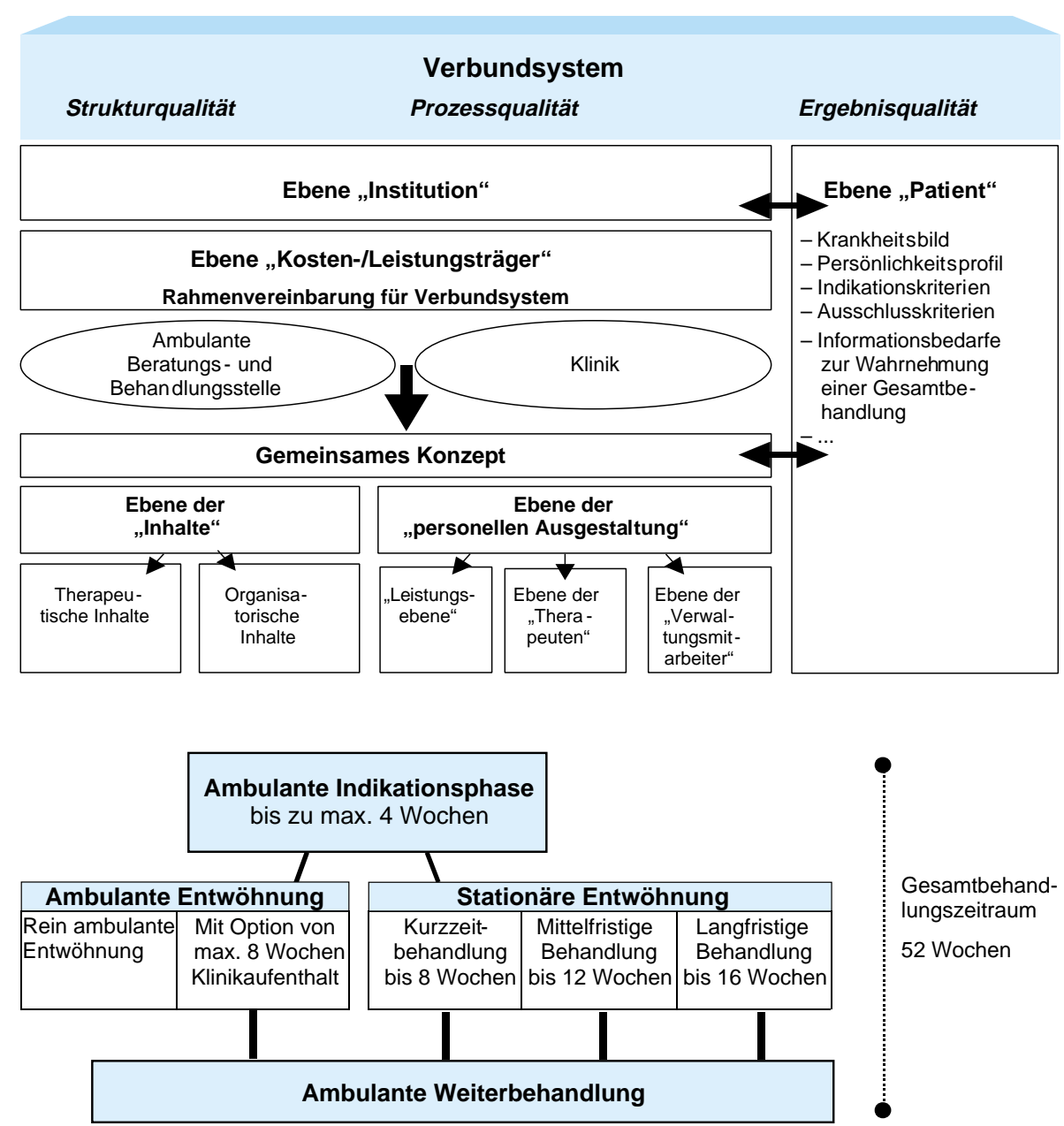

Abb. 1 Ebenen eines Verbundsystems.

Abb. 2 Behandlungsvarianten in EVS.

\footnotetext{
${ }^{3}$ Das Rahmenkonzept für das Modellprojekt „Entwöhnung im Verbundsystem (EVS)“ [13] in der Fassung vom 5. Oktober 2000 legt den Leistungsrahmen für das Modellvorhaben fest.
}
${ }^{4}$ Das therapeutische Gesamtkonzept lag im Februar 2001 im zweiten Entwurf vor und soll während der Modelllaufzeit von den Beteiligten fortgeschrieben werden. Im Februar/März 2001 wurde die Koopera- tionsvereinbarung als formelle Basis der Zusammenarbeit in diesem trägerübergreifenden Verbund unterzeichnet.


lemstellungen, die sich im Laufe des Projektes ergeben, wie z.B. die Erarbeitung des Rahmenkonzepts, des therapeutischen Gesamtkonzepts, der Kooperationsvereinbarung sowie deren Fortschreibung, werden im Qualitätszirkel erörtert und konstruktiv in den Implementierungsprozess einbezogen. Alle vier bis sechs Wochen dienen die Sitzungen dazu, Informationen über aktuelle Entwicklungen auszutauschen und eingeleitete Veränderungen in überschaubaren Zeiträumen zu überprüfen.

Der Qualitätszirkel bietet als Diskussionsforum den Vorteil, Transparenz über das Vorgehen des jeweils anderen und ein Verständnis für notwendige Entscheidungen zu schaffen.

Innovation „Kooperationsstandards“ - Was beinhaltet gute Kooperation?

Vor dem Beginn der Implementierung eines Verbundmodells ist der Begriff und die Ausgestaltung von Kooperation zentral: Was beinhaltet gute Zusammenarbeit sowohl zwischen ambulanten und stationären Leistungsanbietern als auch zwischen Leistungsanbietern und dem Leistungsträger? Welche Strukturen sind zu schaffen und welche Aspekte zu berücksichtigen? Um diesen Fragen näher auf den Grund zu gehen, bildete für das Modellprojekt EVS eine Analyse des „Status quo“, d. h. bereits bestehender und erprobter Kooperationsmodelle im Suchtkrankenhilfebereich, die Grundlage für die Entwicklung so genannter Kooperationsstandards. Aus der eingehenden Beschäftigung mit vorhandenen Modellen konnten Probleme und Schwachstellen aufgedeckt werden, denen im Modellprojekt EVS begegnet werden soll. Die Analyse des Status quo führte im Weiteren zu einer Ergänzung derjenigen Aspekte, die sich für die Schaffung sinnvoller und funktionierender Kooperationsbeziehungen als wichtig erwiesen haben. Sie sind in einem Kriterienkatalog für „gute Kooperation“, der als eine Art „Reflektionsfolie“ zur Planung, Implementierung und Weiterentwicklung von Verbundmodellen dienen kann, zusammengestellt [13]. Im Rahmen der Modellumsetzung bedürfen diese Kriterien der stetigen Überprüfung, Umsetzung und Fortschreibung.

\section{Resümee aus den Erfahrungen bisheriger Modellprojekte}

Die zur Analyse ausgewählten regionalen Kooperationsmodelle im Suchtkrankenhilfebereich beziehen sich auf die Vernetzungsstrukturen zwischen den drei zu unterscheidenden Versorgungssektoren (Suchtkrankenhilfe, psychosoziale Versorgung und medizinische Basisversorgung [14]). Zu nennen sind hier das seit 1978 laufende „Tübinger Modell: Kombination stationärer und ambulanter Therapie“ [5], das von 1988 bis 1992 angelegte „Modellprojekt Intervalltherapie bei Alkohol- und Medikamentenabhängigkeit“ [6], das seit 1992 ins Leben gerufene „Modellprojekt Kassel: Tagesklinik für Suchtkranke des psychiatrischen Krankenhauses Merxhausen“" [15,16], das seit 1995 geplante und 1996 implementierte „Modellprojekt Nachgehende Sozialarbeit“ [17] und das seit 1998 existierende „Modellprojekt in der Pfalz: Integrierte stationär-ambulante Rehabilitation (ISAR)“ $[18,19]$.

An dieser Stelle soll nicht auf die Einzelheiten der verschiedenen Projekte eingegangen, sondern vielmehr eine bewertende $\mathrm{Zu}$ sammenfassung hinsichtlich der für Kooperation relevanten Gesichtspunkte angestrebt werden.
Aus den Kritikpunkten ergeben sich mehrere für Kooperation zu berücksichtigende Dimensionen, die der Klärung bedürfen und die folgende grundsätzliche Fragestellung berühren: „Wer arbeitet mit wem, wie, zu welchem Zweck, mit welchem Ziel und mit welchem Erfolg zusammen?“ [21: 74]. Das bedeutet, es müssen verbindliche Aussagen zu der Anzahl der beteiligten Institutionen, zu gemeinsamen Zielen, Bereichen und Modalitäten der Kooperation (z. B. regionale, direkte Zusammenarbeit) sowie zu den Erfolgsmaßen gemacht werden. Der Erfolg bzw. die Qualität vernetzter Hilfemaßnahmen im Rehabilitationssystem sollte sich auf den von Donabedian [22] eingeführten Ebenen Struktur-, Prozess- und Ergebnisqualität abbilden lassen, wie es in der Darstellung der in Tab. 1 genannten Schlussfolgerungen bereits angestrebt wurde. Nur so kann ein derartig komplexes Gebilde wie Kooperation, sowohl im Hinblick auf zu schaffende Rahmenbedingungen (z.B. gemeinsame Kooperationsgremien, gemeinsames Konzept), die in diesen Strukturen stattfindenden Arbeitsabläufe (z. B. wechselseitige Information über die Patienten/Klienten) als auch die Resultate (z. B. Behandlungserfolg) beurteilt werden.

\section{Die Matrix „Kooperationsstandards zur Entwicklung, Analyse und Bewertung von Kooperation"}

Die im Kontext dieses Modellprojekts vorgestellte Kooperationskriterien-Matrix orientiert sich an den Kooperationsbeteiligten und gliedert das noch weiter zu strukturierende Feld in die drei übergeordneten Ebenen:

\section{Ebene Institution (Leistungsanbieter)}

\section{Ebene Patient/Klient}

\section{Ebene Kosten-/Leistungsträger}

In den drei Ebenen sollen die je nach Beteiligten spezifischen Ausgangsgegebenheiten, die mit in die Kooperation gebracht werden (vgl. Abb.1: Ebenen eines Verbundsystems), abgebildet und berücksichtigt werden. Die Aufgliederung der Kooperationskriterien betreffend der Struktur-, Prozess- und Ergebnisqualität geschieht hier aus analytischen Gründen; es ist selbstverständlich, dass sich ein derart komplexes Phänomen wie Kooperation in der alltäglichen Praxis nicht immer differenziert aufsplitten lässt, was die Identifizierung und kurzfristige Bewältigung vorhandener Problembereiche erschweren kann. Trotz analytischer Trennung innerhalb der Matrix wird diesem Verständnis des Ineinandergreifens verschiedener Kooperationsaspekte mit Hilfe entsprechender Querverweise zu genügen versucht.

Ein Transfer dieser Kooperationssystematik auf bestehende Kooperationsmodelle zeigt diverse Schwachstellen, die hier aus Platzgründen nicht aufgeführt werden können, aber neben den eingangs erwähnten Defiziten v.a. die Bereiche „Informationsfluss zwischen Leistungsanbietern und Leistungsträgern“, „Klima der persönlichen Beziehung der Mitwirkenden“, „Offenlegung der finanziellen Ausgestaltung für Kooperationsaktivitäten“ sowie „Verwaltungstechnische Probleme (z. B. Transparenz über das Antragsverfahren oder die kurzfristige Bereitstellung von Klinikplätzen bei voller Auslastung)“ betreffen. 
Tab. 1 Wichtige Einflussfaktoren für Kooperationsmodelle

\section{Grundlagen:}

- Kooperationsmodelle sollten Fragen nach der Leitung, der Trägerschaft der beteiligten Einrichtungen sowie der regionalen vs. überregionalen Vernetzung berücksichtigen.

- Die Wohnortnähe der ambulanten Einrichtung muss gewährleistet sein, das bedeutet eine Erreichbarkeit mit öffentlichen Verkehrsmitteln innerhalb von 45 bis maximal 60 Minuten.

\section{Auf der Strukturebene:}

- Die Therapiekonzepte müssen in sich schlüssig und realisierbar aufgebaut sein und die Kooperationsschritte nachvollziehbar darstellen (z. B. in einem Gesamtkonzept).

- Elemente von Qualitätsmanagement sollten vorhanden sein.

- Während der Gesamtmaßnahmen sollten unterschiedliche inhaltliche Schwerpunkte in den therapeutischen Maßnahmen zwischen ambulanten und stationären Leistungsanbietern gesetzt werden.

- Das Aufzeigen und Vermitteln von materiellen und immateriellen Hilfen sind zentrale Aufgaben der Fachkräfte in den Beratungsstellen (Einsatz von CaseManagern, Koordinatoren).

\section{Auf der Prozessebene:}

- Der klientenbezogene Informationsfluss zwischen den Einrichtungen ist zur Gewährleistung therapeutischer Konstanz herzustellen. Eine gemeinsame Behandlungsplanung sollte erfolgen; besondere Aufmerksamkeit ist der Schnittstellengestaltung in den ambulant-stationären Übergängen zu schenken.

- Ein gemeinsames bzw. abgestimmtes Begriffsverständnis (Diagnostik, Behandlungsdokumentation) sollte Grundlage der gemeinsamen Arbeit sein.

- Verbindliche Aufgaben/Zuständigkeiten im arbeitsteiligen Gesamtbehandlungsprozess sollten festgelegt werden.

- Die im Allgemeinen praktizierte „Komm-Struktur“ hat sich als nachteilig erwiesen; mehr aufsuchende Arbeit ist erforderlich (z. B. persönlicher Besuch bei Versäumnis der ambulanten Weiterbehandlung).

- Die Information des Klienten/Patienten über den Gesamtbehandlungsverlauf muss frühzeitig erfolgen und ausreichende Kenntnis über die Arbeit der Kooperationspartner widerspiegeln.

- Durch entsprechende Öffentlichkeitsarbeit sollten sich die Verbundeinrichtungen über aktuelle Konzepte, Veränderungen, Vorgehensweisen usw. informieren. Auf der Ergebnisebene:

- Die Behandlungsschritte und -erfolge sollten in einer fortlaufenden Dokumentation transparent gemacht werden.

[20: 7, 11: 32, 9]

Man kann resümieren, dass Kooperationsvorhaben von vielschichtigen Einflussfaktoren bestimmt werden, die nur schwerlich alle Berücksichtigung finden können. Die Zusammenschau dieser Faktoren in einem Kriterienkatalog kann jedoch im Vorfeld der Implementierung von Kooperationsmodellen dazu dienen, möglichst viele potentielle Problembereiche aufzudecken, bevorstehende Organisationsfelder und Aufgaben zu antizipieren und die Ergebnisse dieses Reflektionsprozesses in die Planung und Umsetzung von Kooperationsanliegen einzubeziehen.

\section{Bisherige Ergebnisse und Auswirkungen des Modellprojekts} EVS - Ausblick

Nach einem halbjährigen Planungs- und Implementierungsprozess werden seit Januar 2001 die ersten Patienten im Modellprojekt EVS behandelt. In den ersten sechs Monaten mussten Wege gefunden und bereitet werden, die eine Vernetzungspraxis nach den hier vorgestellten Maßstäben begünstigen helfen. In dem bereits angesprochenen Gesamtkonzept aller beteiligten Leistungsanbieter werden u. a. die theoretischen Behandlungsgrundlagen, Profile der beteiligten Einrichtungen, Ziele und Methoden sowie Inhalte der Entwöhnungsbehandlung nach ambulanten und stationären Schwerpunkten abgebildet. Aspekte der Kooperation werden dabei besonders herausgestellt. Klare Regelungen betreffen die gezielte Vorbereitung auf den Klinikaufenthalt mit Hilfe einer speziellen ambulanten Vorlaufsphase (Indikationsphase), persönliche Übergabegespräche in den Übergängen der Behandlungssettings unter Teilnahme des Patienten und regelmäßige Fallbesprechungen zur Abstimmung der Therapieplanung. Der Patienteninformation sowie Behandlungsdokumenta- tion (auch gegenüber dem Leistungsträger) wird spezielle Aufmerksamkeit geschenkt.

In einem Rahmenkonzept (siehe oben) ist der Leistungsrahmen, der fünf möglichen Behandlungsvarianten in einem Zeitraum bis zu einem Jahr vorsieht, sowie das Antragsverfahren transparent dargestellt worden (vgl. Abb. 2).

In EVS beginnt die Behandlung grundsätzlich ambulant mit der Indikationsphase. Je nach individuellem Behandlungsbedarf können nachfolgend stationäre Phasen unterschiedlicher Dauer in Anspruch genommen werden. Die aufgeführten stationären Therapiezeiten sind jeweils Maximalzeiträume, deren Ausschöpfung anhand der Behandlungsvoraussetzungen des Patienten genau überprüft werden sollten. Die besonders hervorzuhebende Möglichkeit flexibler, auch mehrmaliger Wechsel zwischen ambulanten und stationären Behandlungsabschnitten ist dann möglich, wenn bei einem (ersten) Klinikaufenthalt nicht die gesamte beantragte Behandlungszeit eingesetzt wird. Die Rehabilitation wird nach einem Klinikaufenthalt mit der ambulanten Weiterbehandlung fortgesetzt und letztendlich abgeschlossen. Ebenfalls können rein ambulante Behandlungen durchgeführt werden.

Neben den Behandlungsvarianten kann als weiteres Novum des Leistungsrahmens in EVS die gesonderte Vergütung von zwei persönlichen Übergabegesprächen (mit jeweils zwei ambulanten Therapieeinheiten bzw. 170 DM plus Fahrtkosten) genannt werden. 
Tab. 2 Matrix „Kooperationsstandards“ als Rahmenschema für die Etablierung von Kooperationsmodellen

I Ebene Institution (Leistungsanbieter)

\author{
1. Grundlagen \\ 1.1 Art und Anzahl der beteiligten Institutionen (z. B. flexible vs. geschlossene Struktur) \\ 1.2 Trägerschaft \\ 1.3 Kooperationsbereiche \\ 1.4 Kooperationsziele (Organisationsziele) $\rightarrow$ zu klientenorientierten Zielen siehe I Ebene 2.4.5 \\ 1.5 Modalitäten der Kooperation \\ 1.5.1 Dauer und Stabilität (z. B. längerfristig, kontinuierlich) \\ 1.5.2 Grad der Formalisiertheit (z. B. Vertrag) \\ 1.5.3 Räumliche Distanz (regional versus überregional) \\ 1.5.4 Unmittelbarkeit (direkte versus indirekte Kontakte) $\rightarrow$ siehe Informationsfluss \\ 1.5.5 Klima der persönlichen Beziehung der Mitwirkenden $\rightarrow$ siehe Informationsfluss
}

2. Strukturelle Ebene

2.1 Organisatorische Inhalte

2.1.1 Organisationsplan (Strukturskizze der Verbundpartner)

2.1.2 Leitung/Koordinationsstelle der Kooperation (z. B. Qualitätszirkel)

2.1.3 Qualitätsmanagement

2.1.4 Gemeinsame Ressourcen (z. B. Räumlichkeiten, Fortbildungen, Supervision)

2.1.5 Umstrukturierung/Neuerschließung von Ressourcen (z. B. Etablierung neuer Einrichtungen)

2.2 Personelle Ausgestaltung

2.2.1 Funktionsträger (z. B. Case-Manager, Koordinator, Qualitätsmanager)

2.2.2 Tätigkeitsprofile/Transparenz über die Arbeitsweise der Mitarbeiter

2.2.3 Personelle Überschneidungen (z. B. Kontinuität des Therapeuten)

2.3 Finanzielle Ausgestaltung (Probleme wie z. B. finanz. Aufwendungen für Kooperationsaktivitäten)

2.4 Gemeinsames Konzept

2.4.1 Patienten-/Klientenbild

2.4.2 Einschluss-/Ausschlusskriterien

2.4.3 Indikationskriterien zu einer Behandlung $\rightarrow$ siehe auch Ebene Patient/Klient

2.4.4 Behandlungsablauf (z. B. Intervalle)

2.4.5 Gemeinsame Ziele/Inhalte

2.4.6 Unterschiedliche Schwerpunktsetzungen in der Behandlung $\rightarrow$ siehe auch Aufgabenverteilung

2.4.7 Angaben zur Behandlungsform (z. B. offene Gruppe, Einzelgespräche)

3. Prozessebene

3.1 Systematischer Informationsfluss

3.1.1 Form (z. B. Arbeitstreffen, Telefonate)

3.1.2 Standards bezüglich Diagnostik und Dokumentation/Informationsträger $\rightarrow$ siehe auch I Ebene 4.1

3.1.3 Häufigkeit

3.1.4 Rechtzeitigkeit/Feedback

3.1.5 Inhalt (z. B. organisatorisches, fallbezogenes, fachliches)

3.2 Aufnahme-/Entlassverfahren

3.2.1 Angaben zur Gewährleistung zur Kontinuität des Therapieverlaufs

3.2.2 Maßnahmen zur Vorbereitung auf die stationäre Phase

3.2.3 Maßnahmen zur Vorbereitung auf die ambulante Weiterbehandlung

3.2.4 Möglichkeiten der Aufrechterhaltung des Kontaktes nach Therapieende

3.3 Aufgabenverteilung

3.3.1 Verbindliche Zuständigkeiten $\rightarrow$ siehe auch unterschiedliche Schwerpunktsetzungen

3.3.2 Anteil organisatorischer Tätigkeiten

4. Ergebnisebene

4.1 Dokumentation ambulant-stationärer Behandlungsverläufe (Haltequote, Rückfallquote, soziale Reintegration, Behandlungszufriedenheit)

$\rightarrow$ siehe auch Gliederungspunkt I 3.1.2

4.2 Dokumentation der Qualität der Kooperation (Akzeptanz bei den Kooperationspartnern und bei der Zielgruppe)

$\rightarrow$ siehe auch Gliederungspunkt I 2.1.3 


\section{Persönlichkeitsprofil}

2. Krankheitsbild

3. Bedürfnislage

3.1 Originäre Bedürfnisse im Bereich Abhängigkeit

3.2 Soziale Bedürfnisse

3.3 Medizinische Bedürfnisse

4. Betreuungskonstanz (Berücksichtigung des Informationsbedarfs des Patienten/Klienten)

III Ebene Kosten-/Leistungsträger

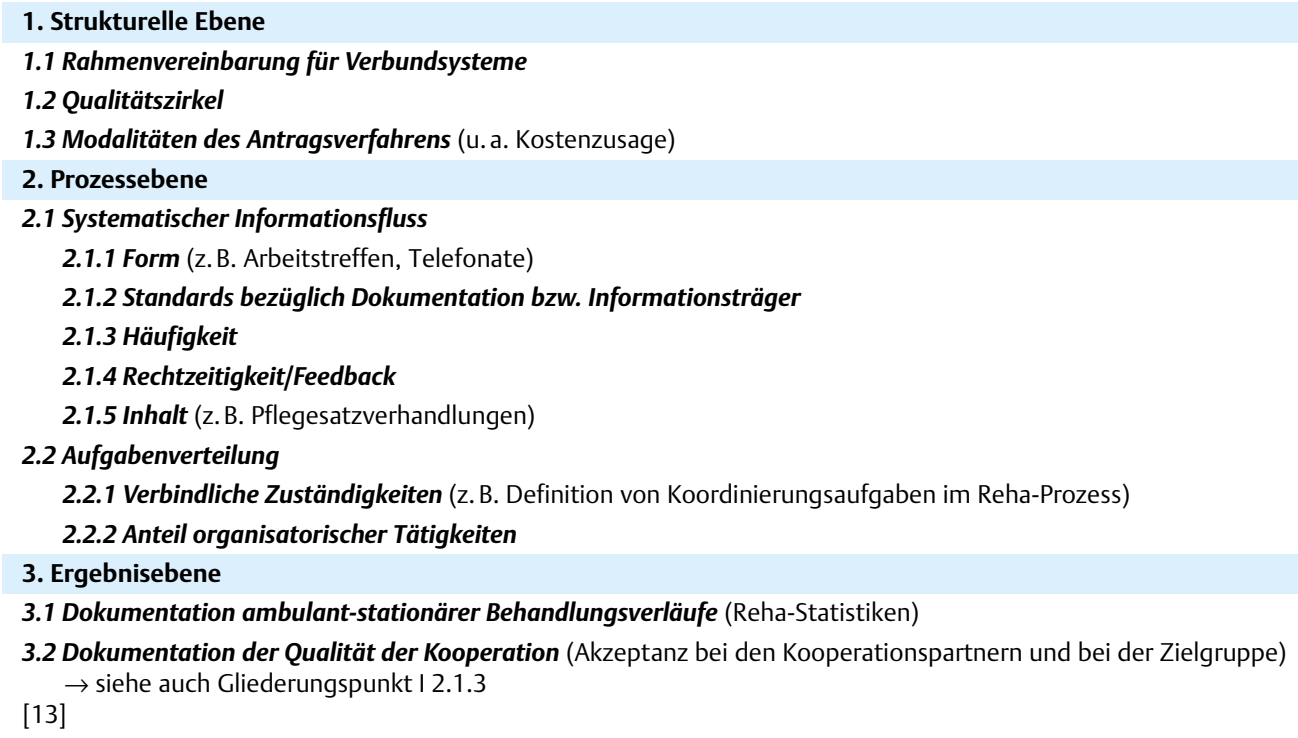

Neben den ja zunächst einmal schriftlichen Veränderungen können aber bereits positive Aspekte in der alltäglichen Praxis hervorgehoben werden. Durch die alle vier bis sechs Wochen stattfindenden Qualitätszirkel sind die Leistungsanbieter untereinander und mit dem Leistungsträger „ein Stück näher zusammengerückt". Die Informationslage über die Arbeit und Probleme der am Verbund beteiligten Einrichtungen konnte gesteigert, die z.T. unterschiedlichen Positionen und Interessen klargestellt werden. Es ist ein Arbeitsklima entstanden, das die zeitnahe Klärung von Fragen zur Durchführung des Modellprojekts begünstigt.

Im Weiteren kann davon berichtet werden, dass innerhalb und unter den beteiligten Einrichtungen ein Kommunikationsprozess in Gang gekommen ist, der sich in der Organisation eines ersten Fachtags zum Modellprojekt mit therapeutischen Mitarbeitern sowie in diversen Informationsveranstaltungen mit den therapeutischen Mitarbeitern der beteiligten Modell- und Kontrolleinrichtungen widerspiegelt.

Für den zukünftigen Verlauf des Projektes stehen noch eine Reihe von Aufgaben an: Es ist z.B. zu klären, inwiefern Grundlagen für eine einheitlichere oder zumindest stärker abgestimmte Diagnostik zwischen ambulanten Beratungsstellen und Fachkliniken zu schaffen sind und die Behandlungsdokumentation in einer fortlaufenden Patientenakte organisiert werden kann. Außerdem muss geprüft werden, inwiefern der Mehraufwand für koordinierende und hilfevermittelnde Tätigkeiten von den Bezugstherapeuten aufgefangen werden kann, oder ob nicht langfristig die Schaffung weiterer personeller Ressourcen in Form von Case-Managern oder Koordinatoren für das reibungslose Funktionieren eines Verbundsystems unabdingbar ist.

\section{Literatur}

${ }^{1}$ Göpel E. Einführung. In: Gesundheitsakademie/Landesinstitut für Schule und Weiterbildung, NRW (Hrsg). Macht - Vernetzung - Gesund? Strategien und Erfahrungen regionaler Vernetzungen im Gesundheitsbereich. Frankfurt am Main: Mabuse 1996; 7-11

${ }^{2}$ Suchtkrankenhilfe im Verbund: Eine kritische Bestandsaufnahme. In: Deutsche Hauptstelle gegen die Suchtgefahren (DHS). Freiburg im Breisgau: Lambertus 1995

${ }^{3}$ Suchtbehandlung: Entscheidungen und Notwendigkeiten. In: Fachverband Sucht e.V. (FVS). Geesthacht: Neuland 1999

${ }^{4}$ Mann K, Batra A. Die gemeindenahe Versorgung von Alkoholabhängigen - Evaluation eines kombinierten stationären und ambulanten Behandlungskonzeptes. Psychiatrische Praxis 1993; 20 (3): 102 - 105

${ }^{5}$ Mundle G, Mann K. Ein Modell zur Integration stationärer und ambulanter Therapie von Alkoholabhängigen. Psycho 1996; 22: 444-451

${ }^{6}$ Ministerium für Kultur, Jugend, Familie und Frauen Rheinland-Pfalz (MSF). Intervalltherapie bei Alkohol- und Medikamentenabhängigkeit. Abschlussbericht des Modellprojekts. Mainz 1994

${ }^{7}$ Meyenberg R, Tielking K, Hedden H. Notwendigkeiten im System der Behandlung von Alkoholabhängigkeit. Ergebnisse einer klinikübergreifenden Studie in Kooperationseinrichtungen einer Landesversicherungsanstalt. In: Fachverband Sucht e.V. (FVS) (Hrsg). Sucht- 
behandlung: Entscheidungen und Notwendigkeiten. Geesthacht: Neuland 1999; 217-234

${ }^{8}$ Neue Wege in der Rehabilitation Alkoholabhängiger - Theoretische und praktische Ansatzpunkte zur Optimierung von Alkoholentwöhnungstherapien. In: Meyenberg R, Tielking K. Oldenburg: BIS-Verlag 1999

${ }^{9}$ Tielking K. Optimierung der Rehabilitation von Alkoholabhängigen. Oldenburg: BIS-Verlag 2000

${ }^{10}$ Böhm M. Verbundsysteme in der Suchtkrankenhilfe. Chancen, Planung und Umsetzung in der Praxis. Kassel: Nicol-Verlag 2000

${ }^{11}$ Standards im Verbundsystem der Suchtkrankenhilfe: Schwerpunkt Drogenarbeit. In: Fachverband Drogen und Rauschmittel e.V. (FDR). Geesthacht: Neuland 1997

${ }^{12}$ Macht - Vernetzung - Gesund? In: Gesundheitsakademie/Landesinstitut für Schule und Weiterbildung, NRW. Frankfurt am Main: Mabuse 1996

13 Tielking K, Kuß G (Hrsg). In: Alkoholentwöhnung im Verbundsystem. Eröffnungsbericht zu einem Modellprojekt in der Region OldenburgBremen. In: Oldenburg: BIS-Verlag 2001

${ }^{14}$ Wienberg G (Hrsg). Die vergessene Mehrheit - zur Realität der Versorgung alkohol- und medikamentenabhängiger Menschen. Bonn: Psychiatrie-Verlag 1992

${ }^{15}$ Ebeling J. Qualitätsmanagement und regionale Kooperation aus Sicht einer Tagesklinik Sucht. In: Deutsche Hauptstelle gegen die Suchtgefahren (Hrsg). Regionale Suchtkrankenversorgung. Konzepte und Kooperationen. Freiburg im Breisgau: Lambertus 1997; 125-136
${ }^{16}$ Engler U, Schlanstedt G. Modellprojekt Kassel: „Tagesklinik für Suchtkranke des psychiatrischen Krankenhauses Merxhausen“. In: Der Bundesminister für Gesundheit (Hrsg). Weiterentwicklung von Hilfen für Alkoholkranke und Menschen mit Alkoholproblemen. Baden-Baden: Nomos-Verl.-Ges 1998; 53-95

${ }^{17}$ Kooperationsmodell nachgehende Sozialarbeit In: FOGS - Gesellschaft für Forschung und Beratung im Gesundheits- und Sozialbereich mbH (Hrsg). Zwischenbericht. Köln 1998

${ }^{18}$ Knauf W, Eichenlaub G. Integrierte stationär-ambulante Rehabilitation - ein Modellprojekt in der Pfalz. Fachverband Sucht e.V.: Suchtbehandlung. Entscheidungen und Notwendigkeiten. Geesthacht: Neuland 1999; $135-146$

19 Janz S, Knauf W, Neeb K. Integrierte stationär-ambulante Rehabilitation (ISAR). Zielsetzung, Organisation und Behandlungsprogramm eines neuen Modells. Vortrag auf der Drogenkonferenz 2000 „Suchtkrankenhilfe gestaltet Veränderungen - Neue Arbeitsweisen und Methoden. Mainz 2000

${ }^{20}$ Ammer F. Stationäre Suchtrehabilitation in Deutschland. Versuch einer Bestandsaufnahme zum Thema Zukunft: Kooperationsmodelle? Sucht Aktuell 1998; 3+4: 7-8

${ }^{21}$ Leune J, Walcker-Meyer C. Suchtkrankenhilfe im Verbund. Eine kritische Bestandsaufnahme. In: Das interne Netz der Suchtkrankenhilfe. In: Deutsche Hauptstelle gegen die Suchtgefahren (DHS). Freiburg im Breisgau: Lambertus 1995; 70-81

${ }^{22}$ Donabedian A. Evaluating the quality of medical care. Milbank Memorial Fund Quarterly 1966; 44: 166-203 